\title{
THE DEGREE OF CONVERGENCE OF A SERIES OF BESSEL FUNCTIONS*
}

BY

\author{
M. G. SCHERBERG
}

A number of problems of mathematical physics require the expansion of an arbitrary function in terms of Bessel functions in a manner analogous to the expansion of such a function in trigonometric functions. An important series in Bessel functions, necessary to the solution of one group of problems, the most familiar of which are the problems of the vibrating circular drumhead and the flow of heat in a cylinder, is the Bessel series, which has the form $\dagger$

$$
f(x)=\sum_{1}^{\infty} B_{n} J_{0}\left(\lambda_{n} x\right)
$$

in which the $B_{n}$ are constants and the $\lambda_{n}$ 's are the positive roots of the equation

$$
l \lambda J_{0}^{\prime}(\lambda)+h J_{0}(\lambda)=0
$$

where either $l=0$ or $h / l>0$.

The more general series to be studied in this paper has the form

$$
f(x)=B(x)+\sum_{1}^{\infty} B_{n} J_{\nu}\left(\lambda_{n} x\right), \quad \nu \geqq 0,
$$

in which the $\lambda$ 's are the positive roots of the equation $\ddagger$

$$
l \lambda J_{\nu}^{\prime}(\lambda)+h J_{\nu}(\lambda)=0
$$

while $B(x)$ is an additional term which is present when (2) has also a pair of imaginary roots $\pm i \lambda_{0}$. Since the functions $x^{1 / 2} J_{\nu}\left(\lambda_{n} x\right)$ form an orthogonal set $\S$ over a range of integration from zero to one, the coefficients $B_{n}$ are found in the usual formal manner and are

$$
B_{n}=\frac{\int_{0}^{1} x f(x) J_{\nu}\left(\lambda_{n} x\right) d x}{\int_{0}^{1} x J_{\nu}^{2}\left(\lambda_{n} x\right) d x} .
$$

The function $B(x) \equiv 0$ when $l=0$ or $h / l+\nu>0$. Otherwise it has a form depending on whether $h / l+\nu$ is equal to or less than zero.\|

* Presented to the Society, September 11, 1931; received by the editors April 25, 1932, and, in revised form, June 20, 1932.

† Watson, Theory of Bessel Functions, 1922, pp. 596-597; Byerly, Fourier's Series, pp. 12-14.

$\ddagger$ The notation $J_{\nu}{ }^{\prime}(x)$ for $(d / d x) J_{\nu}(x)$ will be used through the paper.

$\S$ Gray and Mathews, Treatise on Bessel Functions, 1922, p. 91.

\|l Watson, loc. cit., pp. 596-597. 
That the series converges to the value of the function $f(x)$ under suitable restrictions on the function, and the range of the variable, has been shown by several writers.* The degree of convergence of a series is the order of magnitude of the difference between the function and the first $n$ terms of the series. Thus, if those restrictions are placed upon the function $f(x)$ which insure the convergence of the series to the proper value in a defined range of $x$, the degree of convergence of the series may be calculated as the order of magnitude of the remainder after $n$ terms.

To avoid undue repetition, a convention of symbol is made at this time. $K$ will designate constants independent of $x$ and $n$ and depending only on such fixed quantities as $\nu$, the number of discontinuities in $f^{\prime}(x)$, etc. The function $\theta$ will be any function of any number of variables which is numerically less than one for all values of the variables considered. The notation $\theta_{1}(x)$ will indicate a function which has one for an upper bound and which has a bounded derivative with respect to $x$.

I. The DEgReE OF CONVERGENCE IN THE ABSENCE OF HIGHER DERIVATIVES

LEMмA 1. If $F(x) / x$ has bounded variation in the interval $0 \leqq x \leqq 1$, then

$$
\int_{0}^{1} F(x) J_{\nu}\left(\lambda_{n} x\right) d x=\frac{K \theta\left(\lambda_{n}\right)}{\lambda_{n}^{3 / 2}}
$$

By means of the asymptotic formula $\dagger$

$$
\begin{aligned}
& J_{\nu}(x)=\left(\frac{2}{\pi x}\right)^{1 / 2}\left\{\cos (x-\alpha)+\frac{K \theta(\nu, x)}{x}\right\}, \\
& \alpha=\frac{2 \nu+1}{4} \pi, \quad \nu \geqq 0,
\end{aligned}
$$

on setting $\Phi=F(x) / x$, we have

$$
\int_{0}^{1} F(x) J_{\nu}\left(\lambda_{n} x\right) d x=\left(\frac{2}{\pi \lambda_{n}}\right)^{1 / 2} \int_{0}^{1} \Phi(x) x^{1 / 2} \cos \left(\lambda_{n} x-\alpha\right) d x+\frac{K \theta}{\lambda_{n}^{3 / 2}} .
$$

Since $\Phi=\Phi_{1}(x)-\Phi_{2}(x)$ in which $\Phi_{1}, \Phi_{2}$ are monotone increasing, we may assume without loss in generality that $\Phi$ is also monotone increasing and hence, by the second law of the mean, 605. p. 189.

* C. N. Moore, these Transactions, vol. 12 (1911), pp. 181-200; also Watson, loc. cit., pp. 576-

$\dagger$ Lipschitz, Crelle's Journal, vol. 56 (1859), pp. 193-196; Watson, loc. cit.; C. N. Moore, loc. cit., 


$$
\begin{aligned}
\int_{0}^{1} & \Phi(x) x^{1 / 2} \cos \left(\lambda_{n} x-\alpha\right) d x \\
& =\Phi(+0) \int_{0}^{\xi} x^{1 / 2} \cos \left(\lambda_{n} x-\alpha\right) d x+\Phi(1-0) \int_{\xi}^{1} x^{1 / 2} \cos \left(\lambda_{n} x-\alpha\right) d x \\
& =\frac{K \theta\left(\lambda_{n}\right)}{\lambda_{n}} .
\end{aligned}
$$

LEMMA 2. In the interval $0 \leqq x \leqq 1$ let the function $f(x)$ be absolutely continuous and let $f^{\prime}(x)$ have bounded variation. Then the general coefficient $B_{n}$ of the series (1) may be written as

$$
\frac{(2 \pi)^{1 / 2} \delta_{l} f(1)(-1)^{n}}{\lambda_{n}^{1 / 2}}+\frac{\pi \nu f(0)}{\lambda_{n}}+\frac{K \theta\left(\lambda_{n}\right)}{\lambda_{n}^{3 / 2}}
$$

where

$$
\delta_{l}= \begin{cases}0 & \text { when } l \neq 0 \\ \pm 1 & \text { when } l=0\end{cases}
$$

The treatment in this part of the paper is similar to that employed by C. N. Moore* in a paper on the uniform convergence of a Bessel series.

The denominator of $B_{n}$ may be written $\dagger$

$$
\int_{0}^{1} x J_{\nu}^{2}\left(\lambda_{n} x\right) d x=\frac{1}{2}\left\{J_{\nu}^{2}\left(\lambda_{n}\right)+J_{\nu+1}^{2}\left(\lambda_{n}\right)\right\}-\frac{\nu}{\lambda_{n}} J_{\nu}\left(\lambda_{n}\right) J_{\nu+1}\left(\lambda_{n}\right)
$$

and with the aid of (5) is readily reduced to the form

$$
\int_{0}^{1} x J_{\nu}^{2}\left(\lambda_{n} x\right) d x=\frac{1}{\pi \lambda_{n}}+\frac{K \theta_{1}\left(\lambda_{n}\right)}{\lambda_{n}^{2}} .
$$

By means of (6), $B_{n}$ now assumes the form

$$
\left[\lambda_{n} \pi+K \theta_{1}\left(\lambda_{n}\right)\right] \int_{0}^{1} x f(x) J_{\nu}\left(\lambda_{n} x\right) d x .
$$

On integration by parts with the aid of the recurrence formula

$$
\frac{d}{d x}\left[x^{\nu+1} J_{\nu+1}(x)\right]=x^{\nu+1} J_{\nu}(x),
$$

the integral in (7) becomes

* C. N. Moore, loc. cit., p. 183.

† Byerly, An Elementary Treatise on Fourier Series, 1902, p. 224, formula 12. 


$$
\begin{aligned}
& \int_{0}^{1} x f(x) J_{\nu}\left(\lambda_{n} x\right) d x \\
& =\frac{1}{\lambda_{n}} \int_{0}^{1} \frac{x f(x)}{\left(\lambda_{n} x\right)^{\nu+1}} d\left[\left(\lambda_{n} x\right)^{\nu+1} J_{v+1}\left(\lambda_{n} x\right)\right] \\
& =\frac{f(1) J_{v+1}\left(\lambda_{n}\right)}{\lambda_{n}}-\frac{1}{\lambda_{n}} \int_{0}^{1} x f^{\prime}(x) J_{\nu+1}\left(\lambda_{n} x\right) d x+\frac{1}{\lambda_{n}} \int_{0}^{1} \nu f(x) J_{v+1}\left(\lambda_{n} x\right) d x \\
& =\frac{f(1) J_{v+1}\left(\lambda_{n}\right)}{\lambda_{n}}+\frac{\nu f(0)}{\lambda_{n}} \int_{0}^{1} J_{\nu+1}\left(\lambda_{n} x\right) d x-\frac{1}{\lambda_{n}} \int_{0}^{1} x f^{\prime}(x) J_{v+1}\left(\lambda_{n} x\right) d x \\
& \quad+\frac{\nu}{\lambda_{n}} \int_{0}^{1}[f(x)-f(0)] J_{v+1}\left(\lambda_{n} x\right) d x .
\end{aligned}
$$

If we assume, as we may without loss in generality, that $f^{\prime}(x)$ is $\geqq 0$ and monotone increasing, then $(1 / x)[f(x)-f(0)]$ will be also positive and monotone increasing.

It follows that the last two integrals in the last line of (9) have the form $K \theta\left(\lambda_{n}\right) / \lambda_{n}{ }^{5 / 2}$.

The integral $\int_{0}^{1} J_{v+1}\left(\lambda_{n} x\right) d x$ of (9) may be written

$$
\frac{1}{\lambda_{n}} \int_{0}^{\lambda_{n}} J_{v+1}(x) d x=\frac{1}{\lambda_{n}}\left\{\int_{0}^{\infty} J_{v+1}(x)-\int_{\lambda_{n}}^{\infty} J_{v+1}(x) d x\right\}
$$

since the integrals in question all converge. Although it is not necessary to go beyond the fact that the integral $\int_{0}^{\infty} J_{\nu+1}(x) d x$ is a function of $\nu$ alone, its value one* will be utilized. By means of (5)

$$
\int_{\lambda_{n}}^{\infty} J_{\nu+1}(x) d x=\int_{\lambda_{n}}^{\infty}\left(\frac{2}{\pi x}\right)^{1 / 2} \sin (x-\alpha) d x+K \int_{\lambda_{n}}^{\infty} \frac{\theta_{1}(x) d x}{x^{8 / 2}}=\frac{K \theta\left(\lambda_{n}\right)}{\lambda_{n}^{1 / 2}} .
$$

Thus

$$
\int_{0}^{1} J_{v+1}\left(\lambda_{n} x\right) d x=\frac{1}{\lambda_{n}}+\frac{K \theta\left(\lambda_{n}\right)}{\lambda_{n}^{3 / 2}} .
$$

To complete the proof of Lemma 2, it remains to reduce the term $f(1) J_{v+1}\left(\lambda_{n}\right) / \lambda_{n}$ of (9). A formula for the roots of equation (2) due to Moore $\dagger$ gives

$$
\lambda_{n}=n \pi+q+\frac{K \theta}{n}=K \gamma(n) \cdot n, \quad 1 \leqq \gamma(n) \leqq 2,
$$

* Gray and Mathews, loc. cit., p. 65, Formula 8.

† C. N. Moore, loc. cit., pp. 189-196. 
where

$$
q= \begin{cases}k \pi-\pi / 2+\frac{2 \nu+1}{4} \pi, & l=0, \\ k \pi+\frac{2 \nu+1}{4} \pi, & l \neq 0\end{cases}
$$

and $k$ is an integer, positive, negative or zero.

From (11)

$$
\begin{aligned}
& \sin \left(\lambda_{n}-\alpha\right)=\sin (n \pi+q-\alpha)+\frac{K \theta(n)}{n}, \quad \alpha=\frac{2 \nu+1}{4} \pi, \\
& = \begin{cases} \pm(-1)^{n}+\frac{K \theta(n)}{n}, & l=0 \\
\frac{K \theta(n)}{n}, & l \neq 0,\end{cases}
\end{aligned}
$$

and (5)

$$
J_{\nu+1}\left(\lambda_{n}\right)=\delta_{l}(-1)^{n}\left(\frac{2}{\pi \lambda_{n}}\right)^{1 / 2}+\left(\frac{2}{\pi \lambda_{n}}\right)^{1 / 2} \frac{K \theta}{n}+\frac{K \theta}{\lambda_{n}^{3 / 2}}
$$

in which $\delta_{l}$ is defined as in the statement of Lemma 2. The conclusion of the lemma follows from a combination of the above results.

TheOREM I. Let $f(x)$ be a function such as described in Lemma 2, and, in addition, let the conditions $\delta_{l} f(1)=\nu f(0)=0$ be satisfied. Then

$$
f(x)-S_{n}(x)=\frac{K \theta(n, x)}{n^{1 / 2}}, \quad 0 \leqq x \leqq 1,
$$

where $S_{n}(x)-B(x)$ is the sum of the first $n$ regular terms of the series (1).

It has been shown* that under the conditions of the Lemma 1 , the series will converge to the value of the function in any sub-interval of $0 \leqq x \leqq 1$ having zero as an end point provided $f(x)$ is continuous in this sub-interval and the product $\nu f(0)=0$, and that it will converge to the value of the function in a sub-interval of $0 \leqq x \leqq 1$ having one as an end point if again $f(x)$ is con-

* C. N. Moore, loc. cit., has shown the convergence to $f(x)$ under conditions which insure "closure" and hence the convergence to $f(x)$ under conditions of the lemma follows if there is convergence at all. 
tinuous in this sub-interval and the product $\delta_{l} f(1)=0$. It, therefore, follows that under the conditions of Theorem I the series will converge to $f(x)$ throughout the interval $0 \leqq x \leqq 1$. Further, from Lemma 2 the general term of the series assumes the form

$$
\frac{K \theta\left(\lambda_{n}\right)}{\lambda_{n}^{3 / 2}} J_{\nu}\left(\lambda_{n} x\right)
$$

Since $J_{\nu}\left(\lambda_{n} x\right)$ is uniformly bounded, ${ }^{*}$ this general term may be written $K \theta(n) / n^{3 / 2}$ and the remainder after $n$ terms becomes $K \theta(n) / n^{1 / 2}$.

Theorem II. Let $f(x)$ be a function such as described in Lemma 1. Then in the interval $0 \leqq a \leqq x \leqq b \leqq 1$

$$
f(x)-S_{n}(x)=\frac{K \theta(x, n)}{n x^{1 / 2}}+\frac{K \delta_{l} f(1) \theta(n, x)}{(1-x) n^{1 / 2}}+\frac{K \theta(n, x)}{x^{3 / 2} n^{2}}+\frac{\nu f(0) K \theta(x, n)}{x^{3 / 2} n^{3 / 2}}
$$

where $a \neq 0$ unless $\nu f(0)=0, b \neq 1$ unless $\delta_{l} f(1)=0$, and

$$
\frac{K \theta(n, x)}{n x^{1 / 2}}=\frac{K \theta(n, x)}{x^{3 / 2} n^{2}} \equiv 0 \text { when } a=0 .
$$

Under the conditions of Lemma 2, the series (1) becomes $\dagger$

$$
\begin{gathered}
\delta_{l} f(1) \sum_{m=n+1}^{\infty} \frac{(-1)^{m} J_{\nu}\left(\lambda_{m} x\right)}{\lambda_{m}^{1 / 2}}+\pi \nu f(0) \sum_{m=n+1}^{\infty} \frac{(-1)^{m} J_{\nu}\left(\lambda_{n} x\right)}{\lambda_{m}} \\
+\sum_{m=n+1}^{\infty} \frac{K \theta\left(\lambda_{m}\right)}{\lambda_{m}^{3 / 2}} J_{\nu}\left(\lambda_{m} x\right) .
\end{gathered}
$$

By means of (5) the general term of the last sum becomes

$$
\frac{K \theta\left(\lambda_{m}\right) \cos \left(\lambda_{m} x-\alpha\right)}{\lambda_{m}^{2} x^{1 / 2}}+\frac{K \theta\left(\lambda_{m}\right)}{x^{3 / 2} \lambda_{m}^{3 / 2}}
$$

which with the aid of (11) yields

$$
\sum_{m=n+1}^{\infty} \frac{K \theta\left(\lambda_{m}\right)}{\lambda_{m}^{3 / 2}} J_{\nu}\left(\lambda_{m} x\right)=\frac{K \theta(n, x)}{n x^{1 / 2}}+\frac{K \theta(n, x)}{n^{2} x^{3 / 2}}, \quad 0 \leqq x \leqq 1 .
$$

The first sum of (13) is zero when $\nu>0$ and $x=0$ since $J_{\nu}(0)=0$. On the other hand if $\nu=0$, then $J_{\nu}(0)=1$, and this sum is that of an alternating

* Watson, loc. cit., p. 44.

$\dagger$ The convergence of the separated parts will be apparent in what follows. 
series of numerically decreasing terms. It has a value, then, which is numerically less than the first term or $K \theta(n) /(n+1)^{1 / 2}$. When $x>0$ the summation is made in three parts.

Let $r$ and $s$ be the smallest integers greater than $(n-1)$ which satisfy the inequalities $\lambda_{r+1} x>k_{1}$ and $\lambda_{s+1} x>k_{2}$ where $k_{1}$ and $k_{2}$ are the first and second positive roots of $J_{r}^{\prime}(x)=0$. Now when $x$ is small $r$ and $s$ will surely be larger than $(n+1)$ and this sum may be divided into three parts in the first two of which $J_{v}\left(\lambda_{n} x\right)$ is monotone. With the arguments of the $\sum$ 's omitted, they are

$$
\sum_{n+1}^{r}+\sum_{r+1}^{s}+\sum_{s+1}^{\infty}=\sigma_{1}+\sigma_{2}+\sigma_{3}
$$

By means of (5)

$$
\begin{aligned}
\sigma_{3} & =\left(\frac{2}{\pi x}\right)^{1 / 2}\left[\sum_{m=s+1}^{\infty} \frac{(-1)^{m} \cos \left(\lambda_{m} x-\alpha\right)}{\lambda_{m}}+\frac{1}{x} \sum_{m=s+1}^{\infty} \frac{(-1)^{m} \theta_{1}\left(\lambda_{m} x\right)}{\lambda_{m}{ }^{2}}\right] \\
& =\sigma_{4}+\sigma_{5} .
\end{aligned}
$$

The sum $\sigma_{5}$ converges absolutely, hence

$$
\left(\frac{\pi}{2}\right)^{1 / 2} \sigma_{5}= \pm x^{-3 / 2} \sum_{k=8+1,8+3, \cdots}\left\{\frac{\theta_{1}\left(\lambda_{k} x\right)}{\lambda_{k}^{2}}-\frac{\theta_{1}\left(\lambda_{k+1} x\right)}{\lambda_{k+1}^{2}}\right\} .
$$

But

$$
\frac{\theta_{1}\left(\lambda_{k} x\right)}{\lambda_{k}^{2}}-\frac{\theta_{1}\left(\lambda_{k+1} x\right)}{\lambda_{k+1}^{2}}=\frac{\theta_{1}\left(\lambda_{k} x\right)-\theta_{1}\left(\lambda_{k+1} x\right)}{\lambda_{k}^{2}}+\theta_{1}\left(\lambda_{k+1} x\right)\left[\frac{1}{\lambda_{k}^{2}}-\frac{1}{\lambda_{k+1}^{2}}\right]
$$

in which by means of (11)

$$
\begin{aligned}
\theta_{1}\left(\lambda_{k} x\right)-\theta_{1}\left(\lambda_{k+1} x\right) & =\left(\lambda_{k+1}-\lambda_{k}\right) x \theta_{1}^{\prime}(\bar{x}), \quad \lambda_{k} x<\bar{x}<\lambda_{k+1} x \\
& =K \theta(k) x .
\end{aligned}
$$

Thus

$$
\begin{aligned}
\sigma_{5} & =\sum_{m=s+1, s+3, \cdots} \frac{K \theta(m)}{x^{1 / 2} \lambda_{m}^{2}}+x^{-3 / 2} \sum_{m=s+1, s+3, \cdots} \theta_{1}\left(\lambda_{m+1} x\right)\left[\frac{1}{\lambda_{k}^{2}}-\frac{1}{\lambda_{k+1}^{2}}\right] \\
& =\frac{K \theta(s+1)}{x^{1 / 2} \lambda_{s+1}}+\frac{K \theta(s+1)}{x^{3 / 2} \lambda_{s+1}^{2}}=\frac{K \theta(n+1)}{n^{1 / 2}}, \quad \lambda_{n} x>k_{2} .
\end{aligned}
$$

The sum $\sigma_{4}$ remains to be treated. By means of (11) one readily finds

$$
\cos \left(\lambda_{m} x-\alpha\right)=\cos [(m \pi+q) x-\alpha]+\frac{K \theta x}{m}
$$


and

$$
\frac{1}{\lambda_{m}}-\frac{1}{m \pi}=\frac{K \theta(m)}{m^{2}}
$$

Hence

$$
\begin{aligned}
\left(\frac{2}{\pi x}\right)^{1 / 2} & \sum_{m=s+1}^{\infty} \frac{(-1)^{m} \cos \left(\lambda_{m} x-\alpha\right)}{\lambda_{m}} \\
= & \left(\frac{2}{\pi x}\right)^{1 / 2} \sum_{m=s+1}^{\infty} \frac{(-1)^{m} \cos [(m \pi+q) x-\alpha]}{m \pi}+\frac{K \theta(s+1)}{(s+1)^{1 / 2}}
\end{aligned}
$$

since

$$
x \lambda_{m}>k_{2}, \quad m>s .
$$

The sum on the right is a linear combination of the real and imaginary parts of

$$
\left(\frac{2}{\pi x}\right)^{1 / 2} \sum_{m=s+1}^{\infty} \frac{1}{m \pi}(-1)^{m} e^{\pi m i x}=\left(\frac{2}{\pi x}\right)^{1 / 2} \sum_{m=s+1}^{\infty} \frac{1}{m \pi} e^{m \pi i(x-1)} .
$$

If $(x-1)=\phi$,

$$
S_{k}(\phi) \equiv S_{k}=\sum_{m=s+1}^{k} e^{m \pi i \phi}=e^{(s+1) \pi i \phi} \frac{1-e^{(k-s) \pi i \phi}}{1-e^{\pi i \phi}}=\frac{K \theta(k, s)}{\phi},
$$

then, by the classical transformation of Abel,

$$
\begin{aligned}
\sum_{m=s+1}^{\infty} \frac{e^{m \pi i \phi}}{m} & =\frac{1}{s+1} S_{s+1}+\frac{1}{s+2}\left(S_{s+2}-S_{s+1}\right)+\cdots \\
& =S_{s+1}\left(\frac{1}{s+1}-\frac{1}{s+2}\right)+S_{s+2}\left(\frac{1}{s+2}-\frac{1}{s+3}\right)+\cdots=\frac{K \theta(\phi, s)}{s \phi} .
\end{aligned}
$$

Therefore

$$
\left(\frac{2}{\pi x}\right)^{1 / 2} \sum_{m=s+1}^{\infty} \frac{(-1)^{m} e^{m \pi i x}}{m \pi}=\frac{K \theta(s+1, x)}{(1-x)(s+1)^{1 / 2}} .
$$

As has already been pointed out $J_{\nu}\left(\lambda_{n} x\right)$ is monotone in the sums $\sigma_{1}$ and $\sigma_{2}$, and hence they are readily reduced to sums of terms which alternate in sign and decrease numerically. They will each have the form

$$
\frac{K \theta(n)}{n^{1 / 2}} \text {. }
$$


The second sum of (13) may be reduced by the methods employed on the first sum. It is found that

$$
\sum_{m=n+1}^{\infty} \frac{(-1)^{m} J_{\nu}\left(\lambda_{m} x\right)}{\lambda_{m}}=\frac{K \theta(n, x)}{x^{1 / 2} n}+\frac{K \theta(n, x)}{x^{3 / 2} n^{3 / 2}} .
$$

The sum (13) is now readily reduced to the form of Theorem II.

II. The DEgree of CONVERGENCE WHEN Higher DeRIVATIVES OF $f(x)$ EXIST

Just as in the case of other well known expansions, the convergence is more rapid when higher derivatives are present provided other conditions are suitably adjusted. The Bessel series requires, in general, rather strong restrictions at the end points of the interval in which the function is represented.

In view of the great similarity of the procedure in Part II to that of Part I, the results in the former will be merely stated and the detailed proofs left to the reader.

LEMma 3. In the interval $0 \leqq x \leqq 1$, let $f(x)$ and its first $(p-2)$ derivatives be continuous and let $f^{(p-1)}(x)$ be absolutely continuous while $f^{(p)}(x)$ has bounded variation. Then the coefficient $B_{n}$ of $J_{v}\left(\lambda_{n} x\right)$ in the series (1) may be written as

$$
\begin{gathered}
B_{n}=\left[\pi \lambda_{n}+K \theta\left(\lambda_{n}\right)\right]\left\{\sum_{m=0}^{m=p-1} \sum_{s=0}^{s=p-1} \frac{(-1)^{p+m+s-1} f^{(s)}(1) k(m, s, \nu) J_{\nu+m+1}\left(\lambda_{n}\right)}{\lambda_{n}^{m+1}}\right. \\
+\frac{1}{\lambda_{n}^{p}} \int_{0}^{1} J_{\nu+p}\left(\lambda_{n} x\right) \sum_{s=0}^{s=p} \frac{(-1)^{s(s)}(x) k(p, s, \nu)}{x^{p-s-1}} d x
\end{gathered}
$$

where

and

$$
f^{(s)}(x)=\frac{d^{s} f(x)}{d x^{s}}
$$

$k(p, s, v)$

$=\sum_{q=8,1}^{p}\left\{\left[1 /\left(1 \cdot 3 \cdot 5 \cdots\left(q-2+\cos ^{2} \frac{\pi q}{2}\right)\left(\frac{q-1-\cos ^{2} \frac{\pi q}{2}}{2}\right) !\right.\right.\right.$

$\times(p-q) !(q-s) ! s !)]$

$\times\left[1 \cdot 3 \cdot 5 \cdots\left(2 p-q-2+\cos ^{2} \frac{\pi q}{2}\right)\left(p-\frac{q+1+\cos ^{2} \frac{\pi q}{2}}{2}\right) !\right.$

$\times q ! \nu(\nu+1) \cdots(\nu+q-s-1)]\}$ 
in which $\nu(\nu+1) \cdots(\nu+q-s-1)$ and $1 \cdot 3 \cdot 5 \cdots\left(q-2+\cos ^{2}(\pi q / 2)\right)$ are to be replaced by 1 when $q=s, q=1$ respectively; and the notation $q=s, 1$ implies that $q=s$ when $s>0$ and $q=1$ when $s=0$.

The proof of Lemma 3 is readily obtained after simple but lengthy calculations by integrations by parts with the aid of recurrence formula (8).

Lemma 4. Let $f(x)$ be a function such as described in Lemma 3. Suppose, further, that $f(x)$ together with its first $(p-2)$ derivatives vanish at the end points $x=0$ and $x=1$. Then the coefficient $B_{n}$ of the series (1) may be written

$$
\frac{(2 \pi)^{1 / 2}\left(\delta_{l}^{2}-\cos ^{2} \frac{\pi p}{2}\right) f^{(p-1)}(1)(-1)^{n+c}}{\lambda_{n}^{p-1 / 2}}+\frac{\pi R(p, \nu) f^{(p-1)}(0)}{\lambda_{n}^{p}}+\frac{K \theta(n)}{\lambda_{n}^{p+1 / 2}}
$$

where $\delta_{l}$ is defined as in Lemma 2, $c$ is an undetermined integer and $R(p, \nu)$ $=\sum_{s=0}^{s=p-1}(-1)^{s} k(p, s, \nu)(k(p, s, \nu)$ as in Lemma 3).

TheOREM III. Let $f(x)$ be a function such as described in Lemma 4, and, in addition, let the conditions

$$
\delta_{l}^{2}-\cos ^{2}(\pi p / 2) f^{p-1)}(1)=R(p, \nu) f^{(p-1)}(0)=0
$$

be satisfied. Then

$$
f(x)-S_{n}(x)=\frac{K \theta(n, x)}{n^{p-1 / 2}}, \quad 0 \leqq x \leqq 1
$$

THEOREM IV. Let $f(x)$ be a function such as described in Lemma 4. Then in the interval $0 \leqq a \leqq x \leqq b \leqq 1$

$$
\begin{gathered}
f(x)-S_{n}(x)=\frac{K \theta(x, n)}{x^{1 / 2} n^{p}}+\frac{\left(\delta_{l}{ }^{2}-\cos ^{2} \frac{\pi p}{2}\right) f^{(p-1)}(1) \theta(n, x)}{(1-x) n^{p-1 / 2}}+\frac{K \theta(n, x)}{x^{3 / 2} n^{p+1}} \\
+\frac{R(p, \nu) f^{(p-1)}(0) K \theta(n, x)}{x^{3 / 2} n^{p+1 / 2}}
\end{gathered}
$$

in which $a \neq 0$ unless $R(p, \nu) f^{(p-1)}(0)=0, b \neq 1$ unless

$$
\delta_{l}^{2}-\cos ^{2}(\pi p / 2) f^{(p-1)}(1)=0
$$

and

$$
\frac{K \theta(n, x)}{x^{1 / 2} n^{p}}=\frac{K \theta(n, x)}{x^{3 / 2} n^{p+1}} \equiv 0 \quad \text { when } a=0 .
$$




\section{ON THE MAGNITUDE OF THE CONSTANTS}

To make more definite the results of the previous sections, the magnitude of the constants which occur in some of the formulas there used have been computed. Due to the fact that the calculations are rather lengthy and cannot be easily summarized for presentation, these results will be given in an informal manner. It was found for $x \geqq 0$ and $\alpha=(2 \nu+1) \pi / 4$,

$$
\begin{array}{rlrl}
J_{\nu}(x) & =\left(\frac{2}{\pi x}\right)^{1 / 2}\left[\cos (x-\alpha)+\frac{2}{3} \frac{\nu^{2} \theta_{1}(x)}{x}\right], & & (2 x)^{1 / 2} \geqq \nu \geqq 5 / 2, \\
& =\left(\frac{2}{\pi x}\right)^{1 / 2}\left[\cos (x-\alpha)+\frac{\theta_{1}(x)}{4 \cdot 2^{1 / 2} x}\right], & 0 \leqq \nu \leqq \frac{1}{2^{1 / 2}}, x>0, \\
& =\left(\frac{2}{\pi x}\right)^{1 / 2}\left[\cos (x-\alpha)+\frac{2^{1 / 2} \theta_{1}(x)}{x}\right], & & 0 \leqq \nu \leqq 3 / 2, x>0, \\
& =\left(\frac{2}{\pi x}\right)^{1 / 2}\left[\cos (x-\alpha)+\frac{20 \theta_{1}(x)}{3 x}\right], & & 0 \leqq \nu \leqq 5 / 2, x>0,
\end{array}
$$

that the positive roots of $J_{v}(x)$ larger than

$$
\left(\frac{8 \nu^{2}}{3 \pi}-\frac{\pi}{2}\right) \text { when } \nu>5 / 2
$$

and larger than

$$
\left(\frac{80}{3 \pi}-\frac{\pi}{2}\right) \text { when } 0 \leqq \nu \leqq 5 / 2
$$

are given by the formulas

$$
\begin{array}{ll}
\lambda_{n}=\alpha_{1}+(n+k) \pi+\frac{(4 / 3) \nu^{2} \theta}{\alpha_{1}+\pi(n+k)}, & \nu>5 / 2, n \geqq 2, \\
\lambda_{n}=\alpha_{1}+(n+k) \pi+\frac{20 \theta}{3 \alpha_{1}+3 \pi(n+k)}, & 0 \leqq \nu \leqq 5 / 2, n \geqq 2, \\
\alpha_{1}=\alpha+\frac{\pi}{2}, &
\end{array}
$$

where $k$ is an integer not less than $(-3)$ for the first equation, and not less than 0 for the second; that the positive roots of $2 \lambda J_{2}^{\prime}(\lambda)+h J_{\nu}(\lambda)=0$ larger than $4 \bar{K} / \pi-\pi / 2$ are given by the formula

$$
\lambda_{n}=\alpha+(n+k) \pi+\frac{2 \bar{K} \theta}{\alpha+\pi(n+k)}, \quad l \neq 0, \alpha=\frac{2 \nu+1}{4} \pi,
$$

where $k$ is an integer not less than $(-3)$ and 


$$
\bar{K}=\frac{\pi}{2}\left[\frac{|\nu+h / l|}{1}+\frac{2 \cdot 2^{1 / 2}}{3}(\nu+1)^{2}\right], \quad \nu>5 / 2
$$

or $k$ is a positive integer or zero, and

$$
\bar{K}=\frac{\pi}{2}\left[2^{1 / 2}+|\nu+h / l|\right], \quad 0 \leqq \nu \leqq 5 / 2 .
$$

UNTVERSTTY OF MINNESOTA,

MnnNeapours, MnNo. 